ESAIM: PROCEEDINGS AND SURVEYS, September 2014, Vol. 45, p. 475-484

J.-S. Dhersin, Editor

\title{
OPTIMAL DESIGN OF BOUNDARY OBSERVERS FOR THE WAVE EQUATION
}

\author{
Pierre Jounieaux ${ }^{1}$, Yannick Privat ${ }^{2}$ and Emmanuel Trélat ${ }^{3}$
}

\begin{abstract}
In this article, we consider the wave equation on a domain of $\mathbb{R}^{n}$ with Lipschitz boundary. For every observable subset $\Gamma$ of the boundary $\partial \Omega$ (endowed with the usual Hausdorff measure $\mathcal{H}^{n-1}$ on $\partial \Omega$ ), the observability constant provides an account for the quality of the reconstruction in some inverse problem. Our objective is here to determine what is, in some appropriate sense, the best observation domain. After having defined a randomized observability constant, more relevant tan the usual one in applications, we determine the optimal value of this constant over all possible subsets $\Gamma$ of prescribed area $\mathcal{H}^{n-1}(\Gamma)=L \mathcal{H}^{n-1}(\partial \Omega)$, with $L \in(0,1)$, under appropriate spectral assumptions on $\Omega$. We compute the maximizers of a relaxed version of the problem, and then study the existence of an optimal set of particular domains $\Omega$. We then define and study an approximation of the problem with a finite number of modes, showing existence and uniqueness of an optimal set, and provide some numerical simulations.
\end{abstract}

\section{Setting of the Shape optimization Problem}

\subsection{A brief state of the art.}

The literature on the optimal observation or sensor location problems is abundant in engineering applications, where mainly the optimal location of sensors or controllers is investigated. In many contributions, numerical tools are developed to solve a simplified version of the optimal design problem where either the partial differential equation has been replaced with a discrete approximation, or the class of optimal designs is replaced with a compact finite dimensional set (see for example $[1,5,22]$ and $[15]$ where such problems are investigated in a more general setting).

From an engineering point of view, the aim is to optimize the number, the position or the shape of sensors in order to improve the estimation of the state of the system. Fields of applications are very numerous, for example structural acoustics, piezoelectric actuators, or vibration control in mechanical structures just to name a few of them, are interested in such a study.

In this study, we model this issue by maximizing an observability constant with respect to the shape an location of the sensors. We refer to $[21,23]$ for a general presentation of observability inequalities for wave like systems. We adopt here the "randomized" approach proposed in $[18,19]$, where the authors introduce a

\footnotetext{
1 Sorbonne Universités, UPMC Univ Paris 06, CNRS UMR 7598, Laboratoire Jacques-Louis Lions, F-75005, Paris, France (jounieaux@ann.jussieu.fr).

2 CNRS, Sorbonne Universités, UPMC Univ Paris 06, UMR 7598, Laboratoire Jacques-Louis Lions, F-75005, Paris, France (yannick.privat@math.cnrs.fr).

3 Sorbonne Universités, UPMC Univ Paris 06, CNRS UMR 7598, Laboratoire Jacques-Louis Lions, Institut Universitaire de France, F-75005, Paris, France (emmanuel.trelat@upmc.fr).
}

(C) EDP Sciences, SMAI 2014 
notion of initial data average observability, more relevant for practical issues, and investigate the problem of maximizing this constant with respect to the shape of the sensors, in the case of an internal observation.

Let us mention $[6,7]$, where the authors use a spectral point of view leading to a similar optimal design problem. They investigate the problem of determining the best possible shape and position of the support of a damping term in a one-dimensional wave equation. They provide a complete characterization of the solutions of this problem and highlight the so-called spillover phenomenon (proved rigorously in [18]).

\subsection{Boundary observability of the $n$-dimensional wave equation}

Let $T$ be a positive real number and $\Omega$ be a bounded open connected set in $\mathbb{R}^{n}$ having a Lipschitz boundary. Consider the homogeneous wave equation with Dirichlet boundary conditions

$$
\begin{array}{ll}
\frac{\partial^{2} y}{\partial t^{2}}-\Delta y=0 & (t, x) \in(0, T) \times \Omega \\
y(t, x)=0 & (t, x) \in(0, T) \times \partial \Omega \\
y(0, x)=y^{0}(x), \frac{\partial y}{\partial t}(0, x)=y^{1}(x) & x \in \Omega .
\end{array}
$$

It is well known that for every $\left(y^{0}(\cdot), y^{1}(\cdot)\right) \in H_{0}^{1}(\Omega, \mathbb{C}) \times L^{2}(\Omega, \mathbb{C})$, there exists a unique solution $y$ of $(1)$ satisfying $y \in C^{0}\left(0, T ; H_{0}^{1}(\Omega, \mathbb{C})\right) \cap C^{1}\left(0, T ; L^{2}(\Omega, \mathbb{C})\right)$.

We endow the Lipschitz set $\partial \Omega$ with the usual Hausdorff measure $\mathcal{H}^{n-1}$. The notation $\mathcal{H}^{n-1}(\Gamma)$ stands for the surface area of $\Gamma$.

For a given $\mathcal{H}^{n-1}$-measurable subset $\Gamma$ of $\partial \Omega$, consider the observable variable $z_{\Gamma}$ defined by, $\forall(x, t) \in$ $\partial \Omega \times[0, T]$,

$$
z_{\Gamma}(t, x)=\chi_{\Gamma}(x) \frac{\partial y}{\partial \nu}(t, x)
$$

where $\chi_{\Gamma}$ denotes the characteristic function of the set $\Gamma, \nu$ denotes the outward pointing normal vector and $\frac{\partial y}{\partial \nu}$ is the normal derivative of $y$.

The system (1)-(2) is said observable in time $T$ if there exists a positive constant $C$ such that the inequality

$$
C\left\|\left(y^{0}, y^{1}\right)\right\|_{H_{0}^{1}(\Omega, \mathbb{C}) \times L^{2}(\Omega, \mathbb{C})}^{2} \leq \int_{0}^{T} \int_{\Gamma}\left|\frac{\partial y}{\partial \nu}(t, x)\right|^{2} d \mathcal{H}^{n-1} d t,
$$

holds for all $y^{0}(\cdot) \in H_{0}^{1}(\Omega, \mathbb{C})$ and $y^{1}(\cdot) \in L^{2}(\Omega, \mathbb{C})$. In this case, we denote the so-called observability constant by $C_{T}\left(\chi_{\Gamma}\right)$ the largest constant in (3). Note that, within the class of $\mathcal{C}^{\infty}$ domains, this observability inequality holds if $(\Gamma, T)$ satisfies the Geometric Control Condition (GCC) (see [2]). An overview of boundary observability results for wave like equations can be found in [21,23].

\subsection{Modeling of the problem}

For practical purposes in engineering, it is relevant to consider the problem of determining, whenever it exists, the optimal shape and location of sensors. In the sequel, we assume that the surface area of sensors is fixed, equal to $L \mathcal{H}^{n-1}(\partial \Omega)$, with $L \in(0,1)$.

It could seem natural to maximize the observability constant $C_{T}\left(\chi_{\Gamma}\right)$ over all possible subsets $\Gamma$ of $\partial \Omega$ such that $\mathcal{H}^{n-1}(\Gamma)=L \mathcal{H}^{n-1}(\partial \Omega)$. According to $(3)$, the constant $C_{T}\left(\chi_{\Gamma}\right)$ is defined by

$$
C_{T}\left(\chi_{\Gamma}\right)=\inf \left\{\frac{\int_{0}^{T} \int_{\Gamma}\left|\frac{\partial y}{\partial \nu}(t, x)\right|^{2} d \mathcal{H}^{n-1} d t}{\left\|\left(y^{0}, y^{1}\right)\right\|_{H_{0}^{1}(\Omega, \mathbb{C}) \times L^{2}(\Omega, \mathbb{C})}^{2}} \mid\left(y^{0}, y^{1}\right) \in H_{0}^{1}(\Omega, \mathbb{C}) \times L^{2}(\Omega, \mathbb{C}) \backslash\{(0,0)\}\right\} .
$$

Such a criterion appears difficult to handle and this modeling is besides not relevant with respect to practical expectations. Indeed, the usual observability constant is deterministic and gives an account for the worst choice 
of initial data. It is thus pessimistic. In practice, many experiments or measures are carried out. It corresponds in taking into account a huge class of initial data, but not necessarily the whole space $H_{0}^{1}(\Omega, \mathbb{C}) \times L^{2}(\Omega, \mathbb{C})$ in the definition of the observability constant $C_{T}\left(\chi_{\Gamma}\right)$. Such issues have been widely discussed in a recent series of articles $[19,20]$. The authors propose a new average observability concept inspired by [3] and introduce the notion of randomized observability constant. It can be seen as the mean of the worst $L^{2}$-norm of the observation variable among almost every initial data. We adopt this choice of modeling in the sequel and we describe it now.

Randomized observability constant. Let $\left(\phi_{j}\right)_{j \in \mathbb{N}^{*}}$ be a Hilbert basis of $L^{2}(\Omega)$ consisting of eigenfunctions

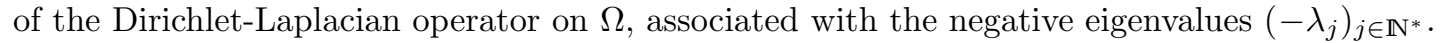

The solution $y$ of (1) can be expanded as

$$
y(t, x)=\sum_{j=1}^{+\infty}\left(a_{j} e^{i \sqrt{\lambda_{j}} t}+b_{j} e^{-i \sqrt{\lambda_{j}} t}\right) \phi_{j}(x)
$$

with

$$
a_{j}=\int_{\Omega} \frac{1}{2}\left(y^{0}(x)-\frac{i}{\sqrt{\lambda_{j}}} y^{1}(x)\right) \phi_{j}(x) d x, \quad b_{j}=\int_{\Omega} \frac{1}{2}\left(y^{0}(x)+\frac{i}{\sqrt{\lambda_{j}}} y^{1}(x)\right) \phi_{j}(x) d x
$$

for every $j \in \mathbb{N}^{*}$.

Using this expression of $y$ and the energy identity

$$
\left\|\left(y^{0}, y^{1}\right)\right\|_{H_{0}^{1}(\Omega, \mathbb{C}) \times L^{2}(\Omega, \mathbb{C})}^{2}=\sum_{j=1}^{+\infty} \lambda_{j}\left(\left|a_{j}\right|^{2}+\left|b_{j}\right|^{2}\right),
$$

the observability constant $C_{T}\left(\chi_{\Gamma}\right)$ can be written as

$$
C_{T}\left(\chi_{\Gamma}\right)=\inf _{\substack{\left(\widetilde{a}_{j}\right),\left(\widetilde{b}_{j}\right) \in \ell^{2}(\mathbb{R}) \\ \sum_{j=1}^{+\infty}\left(\left|\widetilde{a}_{j}\right|^{2}+\left|\widetilde{b}_{j}\right|^{2}\right)=1}} \int_{0}^{T} \int_{\Gamma}\left|\sum_{j=1}^{+\infty}\left(\frac{\widetilde{a}_{j}}{\sqrt{\lambda_{j}}} e^{i \sqrt{\lambda_{j}} t}+\frac{\widetilde{b}_{j}}{\sqrt{\lambda_{j}}} e^{-i \sqrt{\lambda_{j}} t}\right) \frac{\partial \phi_{j}}{\partial \nu}(t, x)\right|^{2} d \mathcal{H}^{n-1} d t
$$

The randomization procedure, introduced in $[18,19]$ consists of replacing $C_{T}\left(\chi_{\Gamma}\right)$ with its randomized counterpart

$$
C_{T, \text { rand }}\left(\chi_{\Gamma}\right)=\inf _{\substack{\left(\widetilde{a}_{j}\right),\left(\widetilde{b}_{j}\right) \in \ell^{2}(\mathbb{R}) \\ \sum_{j=1}^{+\infty}\left(\left|\widetilde{a}_{j}\right|^{2}+\left|\widetilde{b}_{j}\right|^{2}\right)=1}} \int_{A} \int_{0}^{T} \int_{\Gamma}\left|\sum_{j=1}^{+\infty}\left(\beta_{1, j}^{\omega} \widetilde{a}_{j} e^{i \sqrt{\lambda_{j}} t}+\beta_{2, j}^{\omega} \widetilde{b}_{j} e^{-i \sqrt{\lambda_{j}} t}\right) \frac{1}{\sqrt{\lambda_{j}}} \frac{\partial \phi_{j}}{\partial \nu}(x)\right|^{2} d x d t d p_{\omega}
$$

where $\left(\beta_{1, j}^{\omega}\right)_{j \in \mathbb{N}^{*}}$ and $\left(\beta_{2, j}^{\omega}\right)_{j \in \mathbb{N}^{*}}$ denote two sequences of independent random variables on a probability space $(A, \mathbb{P})$ having mean equal to zero, variance equal to 1 and verifying for all $(j, k)$ in $\left(\mathbb{N}^{*}\right)^{2}$, with $j \neq k$,

$$
\mathbb{P}\left(\beta_{1, j}^{\omega}= \pm 1\right)=\mathbb{P}\left(\beta_{2, j}^{\omega}= \pm 1\right)=\frac{1}{2} \quad \text { and } \quad \mathbb{E}\left(\beta_{1, j}^{\omega} \beta_{2, k}^{\omega}\right)=0
$$

The constant $C_{T, \text { rand }}\left(\chi_{\Gamma}\right)$ is the largest constant in the so-called randomized observability inequality

$$
C_{T, \text { rand }}\left(\chi_{\Gamma}\right)\left\|\left(y_{\omega}^{0}, y_{\omega}^{1}\right)\right\|_{H_{0}^{1}(\Omega) \times L^{2}(\Omega)}^{2} \leq \mathbb{E}\left(\int_{0}^{T} \int_{\Gamma}\left|\frac{\partial y_{\omega}}{\partial \nu}(t, x)\right|^{2} d \mathcal{H}^{n-1} d t\right)
$$


holding for all $y^{0}(\cdot) \in H_{0}^{1}(\Omega, \mathbb{C})$ and $y^{1}(\cdot) \in L^{2}(\Omega, \mathbb{C})$, where $y_{\omega}$ denotes the solution of the wave equation with the random initial data $\left(y_{\omega}^{0}, y_{\omega}^{1}\right)$ determined by their Fourier coefficients $a_{j}^{\omega}$ and $b_{j}^{\omega}$ deduced from the Fourier coefficients $a_{j}$ and $b_{j}$ of the initial data $\left(y^{0}, y^{1}\right)$ (see (6) for the explicit dependence between the Fourier coefficients and the initial datum) by $a_{j}^{\omega}=\beta_{1, j}^{\omega} a_{j}$ and $b_{j}^{\omega}=\beta_{1, j}^{\omega} b_{j}$. We have the following result.

Proposition 1. Let $\Gamma$ denote a $\mathcal{H}^{n-1}$-measurable subset of $\partial \Omega$. One has

$$
C_{T, \text { rand }}\left(\chi_{\Gamma}\right)=T \inf _{j \in \mathbb{N}^{*}} \frac{1}{\lambda_{j}} \int_{\partial \Omega} \chi_{\Gamma}(x)\left(\frac{\partial \phi_{j}}{\partial \nu}(x)\right)^{2} d \mathcal{H}^{n-1},
$$

where $\chi_{\Gamma}$ denote the characteristic function of $\Gamma$.

This leads to consider the following optimal design problem, modeling in our view in a relevant way, the problem of optimizing the placement and shape of sensors along the boundary of $\Omega$.

Optimal design problem. We investigate the problem of maximizing

$$
J\left(\chi_{\Gamma}\right)=\inf _{j \in \mathbb{N}^{*}} \frac{1}{\lambda_{j}} \int_{\partial \Omega} \chi_{\Gamma}(x)\left(\frac{\partial \phi_{j}}{\partial \nu}(x)\right)^{2} d \mathcal{H}^{n-1},
$$

over all possible subsets $\Gamma$ of $\partial \Omega$ of Hausdorff measure $\mathcal{H}^{n-1}(\Gamma)=L \mathcal{H}^{n-1}(\partial \Omega)$.

Remark 1. The motivation for maximizing the constant $C_{T, \text { rand }}\left(\chi_{\Gamma}\right)$ instead of the observability constant $C_{T}\left(\chi_{\Gamma}\right)$ has been discussed previously. The issue of maximizing the functional $C_{T}\left(\chi_{\Gamma}\right)$, even if it is not so relevant for practical applications, remains plausible and is intrinsically interesting. Nevertheless, it appears to be very difficult from the theoretical and numerical points of view, due to the presence of crossed terms $\int_{\Gamma} \frac{\partial \phi_{j}}{\partial \nu}(x) \frac{\partial \phi_{k}}{\partial \nu}(x) d \mathcal{H}^{n-1}$ measuring the interaction over $\Gamma$ between distinct eigenfunctions.

\section{Study of the optimal Design Problem (9)}

\subsection{Investigating the optimal value}

Define the admissible set

$$
\mathcal{U}_{L}=\left\{\chi_{\Gamma} \mid \Gamma \text { is a } \mathcal{H}^{n-1} \text {-measurable subset of } \partial \Omega \text { of measure } \mathcal{H}^{n-1}(\Gamma)=L \mathcal{H}^{n-1}(\partial \Omega)\right\} .
$$

Note that the problem (9) is

$$
\sup _{\chi_{\Gamma} \in \mathcal{U}_{L}} J\left(\chi_{\Gamma}\right)
$$

Since the set $\mathcal{U}_{L}$ is not compact for the $L^{\infty}$ weak-star topology, it is usual to introduce a convexified version of the problem (9). For this reason, we consider the convex closure of the set $\mathcal{U}_{L}$,

$$
\overline{\mathcal{U}}_{L}=\left\{a \in L^{\infty}(\partial \Omega ;[0,1]) \mid \int_{\partial \Omega} a(x) d \mathcal{H}^{n-1}=L \mathcal{H}^{n-1}(\partial \Omega)\right\},
$$

and the relaxed formulation of the problem (9),

$$
\sup _{a \in \overline{\mathcal{U}}_{L}} J(a)
$$

where

$$
J(a)=\inf _{j \in \mathbb{N}^{*}} \frac{1}{\lambda_{j}} \int_{\partial \Omega} a(x)\left(\frac{\partial \phi_{j}}{\partial \nu}(x)\right)^{2} d \mathcal{H}^{n-1} .
$$


Proposition 2. Let $L \in(0,1)$. The relaxed problem (12) has at least one solution $a^{*} \in \overline{\mathcal{U}}_{L}$.

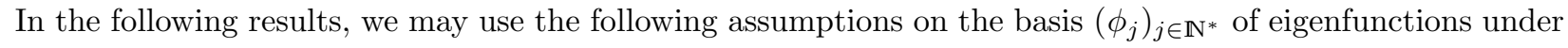
consideration.

Uniform boundedness. There exists $A>0$ such that for every $j \in \mathbb{N}^{*}$

$$
\left\|\frac{\partial \phi_{j}}{\partial \nu}\right\|_{L^{\infty}(\partial \Omega)} \leq A \lambda_{j}
$$

Remark 2. Assumption (14) holds true for whenever $\Omega$ is a square or a disk (see Section 2.2).

Weak Quantum Ergodicity of Boundary values (WQEB) property. There exist a subsequence of $\frac{1}{\lambda_{j}}\left(\frac{\partial \phi_{j}}{\partial \nu}\right)^{2} d \mathcal{H}^{n-1}$ converging vaguely to the uniform measure $\frac{2}{n|\Omega|} d \mathcal{H}^{n-1}$.

Remark 3. It has been proved in [3] that the WQEB property holds true for ergodic piecewise smooth domains $\Omega .^{1}$

Strong Quantum Ergodicity of Boundary values (SQEB) property. The sequence of measures $\frac{1}{\lambda_{j}}\left(\frac{\partial \phi_{j}}{\partial \nu}\right)^{2} d \mathcal{H}^{n-1}$ is converging vaguely to the uniform measure $\frac{2}{n|\Omega|} d \mathcal{H}^{n-1}$.

Remark 4. We insist on the fact that, even if WQEB is satisfied for smooth ergodic domains, very few of them may satisfy the more restrictive SQEB property. For instance in dimension 2 the disk is an example of domain $\Omega$ which satisfies this last property. At the opposite, SQEB does not hold true for the square (however this particular case, studied in section 2.2, satisfies the WQEB property) or general rectangles.

It is clear that, under WQEB and (14) there exists a subsequence of $\frac{1}{\lambda_{j}}\left(\frac{\partial \phi_{j}}{\partial \nu}\right)^{2}$ converging to $\frac{2}{n|\Omega|}$ for the $L^{\infty}(\partial \Omega)$ weak-star topology, then one has $J(a) \leq 2 \frac{L \mathcal{H}^{n-1}(\partial \Omega)}{n|\Omega|}$, for every $a \in \overline{\mathcal{U}}_{L}$.

For every $x_{0} \in \Omega$, define

$$
a_{x_{0}}(x)=\frac{L \mathcal{H}^{n-1}(\partial \Omega)}{n|\Omega|}\left\langle x-x_{0}, \nu\right\rangle
$$

for every $x \in \partial \Omega$, where $\langle\cdot, \cdot\rangle$ denotes the standard Euclidean inner product in $\mathbb{R}^{n}$. Using the instrumental Rellich identity (see for example [21, chap.7, p.232])

$$
2 \lambda_{j}=\int_{\partial \Omega}\left\langle x-x_{0}, \nu\right\rangle\left(\frac{\partial \phi_{j}}{\partial \nu}(x)\right)^{2} d \mathcal{H}^{n-1}
$$

we infer the following result.

Proposition 3. If $\Omega$ satisfies $W Q E B$ and (14), and if there exists $x_{0} \in \Omega$ such that $0 \leq a_{x_{0}}(x) \leq 1$ for every $x \in \partial \Omega$, then $a_{x_{0}}$ is a solution of the relaxed problem (12), and

$$
\max _{a \in \overline{\mathcal{U}}_{L}} J(a)=2 \frac{L \mathcal{H}^{n-1}(\partial \Omega)}{n|\Omega|} .
$$

In the next remark, we provide a class of examples where the existence of $x_{0}$ such that $a_{x_{0}} \in \overline{\mathcal{U}}_{L}$ is true.

\footnotetext{
${ }^{1}$ Actually in these references it is proved that a density one subsequence of $\frac{1}{\lambda_{j}}\left(\frac{\partial \phi_{j}}{\partial \nu}\right)^{2} d \mathcal{H}^{n-1}$ converges to the uniform measure under ergodic assumptions.
} 
Remark 5. Set $x_{0}=0$. Assume that $\Omega$ is convex, and that there exist two balls $B_{e}$ and $B_{i}$ of $\mathbb{R}^{n}$, centered at the origin, of radii $R_{e}$ and $R_{i}$ such that

$$
B_{i} \subset \Omega \subset B_{e} \quad \text { and } \quad\left(\frac{R_{e}}{R_{i}}\right)^{n} \leq \frac{n^{2}}{4 L} .
$$

Then, $a_{0}$ belongs to $\overline{\mathcal{U}}_{L}$.

Indeed, it follows from the convexity of $\Omega$, that $0 \leq\langle x, \nu\rangle \leq R_{e}$ for every $x \in \partial \Omega$. Recall that in the class of convex sets, the volume and the perimeter are monotone increasing for the inclusion. Thus, $\left|B_{i}\right| \leq|\Omega|$ and $\mathcal{H}^{n-1}(\partial \Omega) \leq \mathcal{H}^{n-1}\left(\partial B_{e}\right)$, so that $a_{0} \leq \frac{4 L}{n^{2}}\left(\frac{R_{e}}{R_{i}}\right)^{n} \leq 1 \quad$ on $\partial \Omega$. for every $x \in \Omega$.

Some admissible choices of domains $\Omega$ in dimension 2 for which $a_{0} \in \overline{\mathcal{U}}_{L}$ are drawn in Figure 1. And for some admissible choices of domains $\Omega$, Figure 2 gives the values of $a_{0}$.
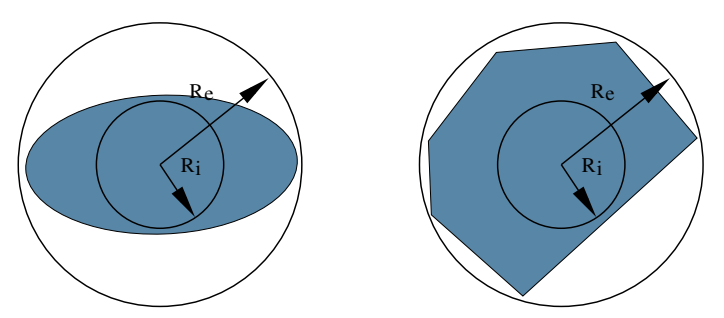

Figure 1. Two domains $\Omega$ in dimension 2 for which $a_{0} \in \overline{\mathcal{U}}_{L}$
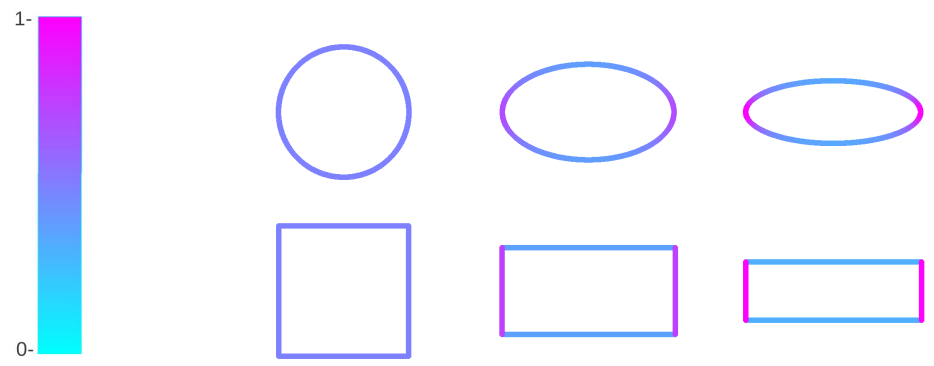

Figure 2. Examples of optimal solutions in $\overline{\mathcal{U}}_{L}$ for several domains $\Omega$

It is clear that $\sup _{\chi_{\Gamma} \in \mathcal{U}_{L}} J\left(\chi_{\Gamma}\right) \leq \sup _{a \in \overline{\mathcal{U}}_{L}} J(a)$. A natural question is to investigate wether this inequality is strict or not. The following theorem states that, under several assumptions it is an equality.

Theorem 1 (No-Gap). If $\Omega$ satisfies $S Q E B$ and (14), and if there exists $x_{0} \in \Omega$ such that $0 \leq a_{x_{0}}(x) \leq 1$ for every $x \in \partial \Omega$, then

$$
\sup _{\chi_{\Gamma} \in \mathcal{U}_{L}} \inf _{j \in \mathbb{N}^{*}} \frac{1}{\lambda_{j}} \int_{\partial \Omega} \chi_{\Gamma}(x)\left(\frac{\partial \phi_{j}}{\partial \nu}(x)\right)^{2} d \mathcal{H}^{n-1}=\sup _{a \in \overline{\mathcal{U}}_{L}} \inf _{j \in \mathbb{N}^{*}} \frac{1}{\lambda_{j}} \int_{\partial \Omega} a(x)\left(\frac{\partial \phi_{j}}{\partial \nu}(x)\right)^{2} d \mathcal{H}^{n-1}
$$

In other words there is no gap between the problem (9) and its convexified version (12).

Corollary 1. Under the assumptions of Theorem 1, we have the optimal value for the problem (9)

$$
\sup _{\chi_{\Gamma} \in \mathcal{U}_{L}} J\left(\chi_{\Gamma}\right)=2 \frac{L \mathcal{H}^{n-1}(\partial \Omega)}{n|\Omega|} .
$$


Now the question is to know if this supremum is reached on $\mathcal{U}_{L}$ or not. In this view we propose in the next section the study of some particular cases.

\subsection{Solving (9) in some particular cases}

In this section, we investigate some particular cases for which SQEB may not hold true (cf remark 4) but however the conclusion holds, highlighting that SQEB is not sharp in Theorem 1.

Proposition 4. Let $L \in[0,1]$.

- Assume that $\Omega=(0, \pi)^{2}$. we have,

$$
\sup _{\chi_{\Gamma} \in \mathcal{U}_{L}} J\left(\chi_{\Gamma}\right)=\max _{a \in \overline{\mathcal{U}}_{L}} J(a)=J(L)=\pi L
$$

Moreover, the supremum over $\mathcal{U}_{L}$ is reached if and only if $\left\{0, \frac{1}{4}, \frac{1}{2}, \frac{3}{4}, 1\right\}$.

- Assume that $\Omega=B(0, R)$. We have,

$$
\sup _{\chi_{\Gamma} \in \mathcal{U}_{L}} J\left(\chi_{\Gamma}\right)=\max _{a \in \overline{\mathcal{U}}_{L}} J(a)=J(L)=\pi R L
$$

Moreover, the supremum over $\mathcal{U}_{L}$ is reached if and only if $L \in\left\{0, \frac{1}{4}, \frac{1}{2}, \frac{3}{4}, 1\right\}$.

We are actually able to characterize explicitly all optimal domains whenever they exist (see [12]). On figures 3 and 4 we provide the drawings of several of them.
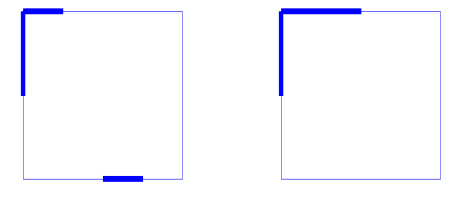

(a) $L=1 / 4$

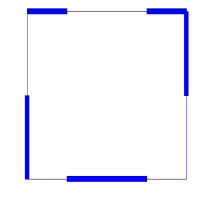

(b) $L=1 / 2$

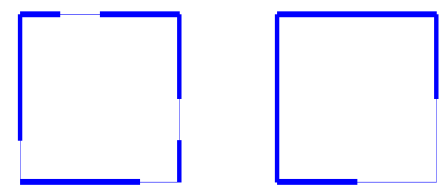

(c ) $L=3 / 4$

FiguRE 3. $\Omega=(0, \pi)^{2}$. Some particular solutions for $L=1 / 4, L=1 / 2$ and $L=3 / 4$.

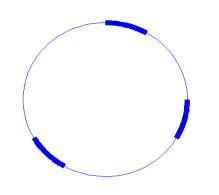

(a) $L=1 / 4$
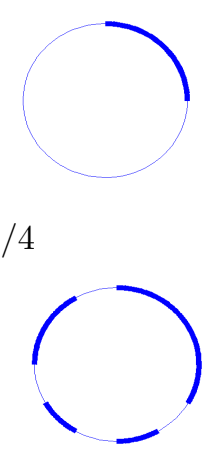
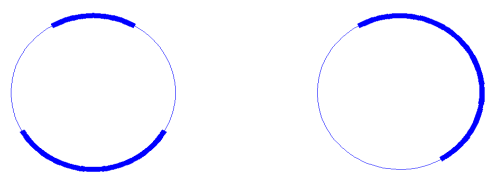

(b) $L=1 / 2$

$$
\text { (c) } L=3 / 4
$$

Figure $4 . \Omega=\left\{x \in \mathbb{R}^{2},\|x\|<1\right\}$. Some particular solutions for $L=1 / 4, L=1 / 2$ and $L=3 / 4$. 


\section{Truncated PROBlem And nUmerical Simulations}

While investigating the optimal value of $J$ on $\mathcal{U}_{L}$, we had to make the two main assumptions SQEB and UB. A natural question now is how to approximate the optimal value when those assumptions are not satisfied. In this view we propose here to truncate the criterion (13) at some order $N \in \mathbb{N}^{*}$, and thus to consider the problem

$$
\sup _{a \in \overline{\mathcal{U}}_{L}} J_{N}(a)
$$

where

$$
J_{N}(a)=\inf _{1 \leq j \leq N} \frac{1}{\lambda_{j}} \int_{\partial \Omega} a(x)\left(\frac{\partial \phi_{j}}{\partial \nu}(x)\right)^{2} d \mathcal{H}^{n-1}
$$

Moreover the study of the truncated problem finds some interest from an engineering point of view, considering that it may be more relevant to take into account only a finite number of modes in this framework. The following $\Gamma$-convergence result states that, when there exists a solution of Problem (17) in $\overline{\mathcal{U}}_{L}$ for every $N \in \mathbb{N}$, then the optimal values of (18) converge to the optimal value of Problem (12). Note that, when the assumptions of Theorem 1 are satisfied, it yields an estimate of the optimal value for the initial problem (9).

Proposition 5. $J_{N} \Gamma$-converges to $J$ in the sense of De Giorgi. Moreover, when there exist a sequence $\left(\chi_{\Gamma_{N}}^{*}\right)_{N \in \mathbb{N}}$ of maximizers of $J_{N}$, then $\chi_{\Gamma_{N}}^{*}$ converges (up to a subsequence) for the $L^{\infty}$ weak-star topology to a maximizer of $J$ in $\overline{\mathcal{U}}_{L}$ and

$$
\max _{a \in \overline{\mathcal{U}}_{L}} J(a)=\lim _{N \rightarrow+\infty} J_{N}\left(\chi_{\Gamma_{N}}^{*}\right)
$$

Remark 6. (1) The sequence $\left(\chi_{\Gamma_{N}}^{*}\right)_{N \in \mathbb{N}^{*}}$ is however not in general a maximizing sequence of $J$. Indeed, using a strategy close to the one used in [7] to emphasize the spillover phenomenon, it is possible to show in the case of the square $\Omega=(0, \pi)^{2}$ that for $\varepsilon>0$ small enough, there exists $N_{0} \in \mathbb{N}^{*}$ such that

$$
J\left(\chi_{\Gamma_{N}}^{*}\right)<\max _{\chi_{\Gamma} \in \mathcal{U}_{L}} J\left(\chi_{\Gamma}\right)-\varepsilon=\frac{L}{2}-\varepsilon
$$

for every $N \geq N_{0}$.

(2) For some particular cases (for instance the square and the ellipse), we have the existence and the uniqueness of a solution for Problem (17) in $\mathcal{U}_{L}$. In the following paragraph we propose some numerical computation of optimal domains for the truncated problem (17) for those particular cases. Moreover, in an ongoing work [12] we prove existence and uniqueness of a solution of Problem (17) under generic assumptions on $\Omega$. This result comes from the fact that for analytic domains, if the functions $\left(\frac{\partial \phi_{j}}{\partial \nu}\right)^{2}$ are independent, one has that a solution must be a level-set of a combination of those functions. This independance holds true under generic assumptions. Anyway, the truncated problem (17) has solutions in $\mathcal{U}_{L}$, for several particular domains such as the square, the disk and the ellipses, computed hereafter.

The following simulations were obtained with a direct method consisting in discretizing the underlying differential equations, the control, and to reduce the optimal control problem to some minimization problem with constraints. We used the code IPOPT (see [24]) combined with AMPL (see [4]) on a standard desktop machine. Figures 5 and 6 , concern the case of the square and the ellipse. The case of the disk computed in Figure 7, is slightly different. In this case we do not have the unicity of a solution in $\mathcal{U}_{L}$. Nevertheless in this case one can find easily which are the optimal domains for Problem (17). Notice that we do not have unicity of the solution and thus propose different examples of solutions. 

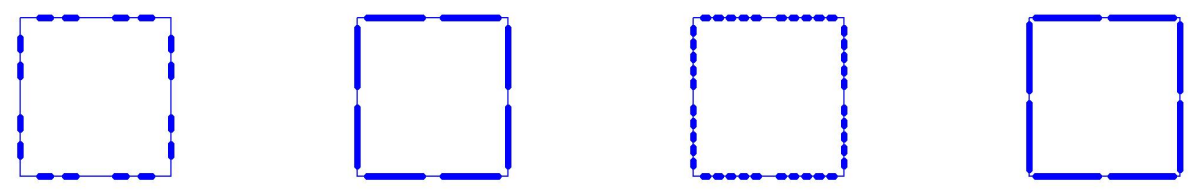

Figure 5. The square for 2 and 5 modes $(L=0.3$, and $L=0.7)$
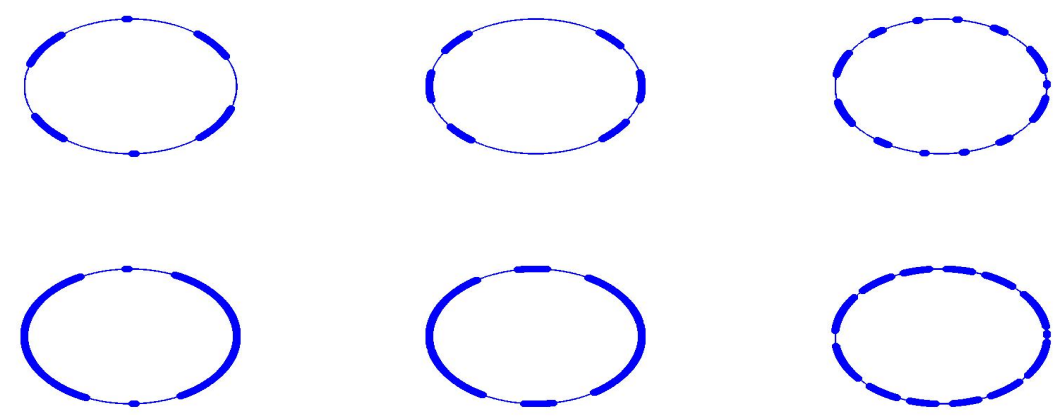

Figure 6 . The ellipse for 5 and 10 and 20 modes $(L=0.3$, and $L=0.7)$
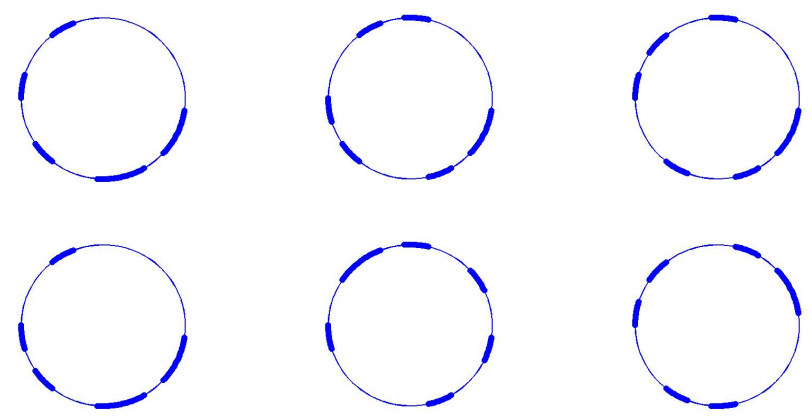

Figure 7. The disk for 1,2 and 5 modes, two examples of solutions $(\mathrm{L}=0.3)$

\section{REFERENCES}

[1] A. Armaoua, M. Demetriou, Optimal actuator/sensor placement for linear parabolic PDEs using spatial $H^{2}$ norm, Chemical Engineering Science 61 (2006), 7351-7367.

[2] C. Bardos, G. Lebeau, J. Rauch, Sharp sufficient conditions for the observation, control, and stabilization of waves from the boundary, SIAM J. Control Optim. 30 (1992), no. 5, 1024-1065.

[3] N. Burq, N. Tzvetkov, Random data Cauchy theory for supercritical wave equations. I. Local theory, Invent. Math. 173 (2008), no. 3, 449-475.

[4] R. Fourer, D.M. Gay, B.W. Kernighan, AMPL: A modeling language for mathematical programming, Duxbury Press, Second edition (2002), 540 pages.

[5] T.J. Harris, J.F. Macgregor, J.D. Wright, Optimal sensor location with an application to a packed bed tubular reactor, AIChE Journal 26 (1980), no. 6, 910-916. 
[6] P. Hébrard, A. Henrot, Optimal shape and position of the actuators for the stabilization of a string, Syst. Cont. Letters 48 (2003), 199-209.

[7] P. Hébrard, A. Henrot, A spillover phenomenon in the optimal location of actuators, SIAM J. Control Optim. 44 (2005), 349-366.

[8] A. Henrot, M. Pierre, Variation et optimisation de formes (French) [Shape variation and optimization] Une analyse géométrique. [A geometric analysis], Math. \& Appl. 48, Springer, Berlin, 2005.

[9] L. F. Ho, Observabilité frontière de l'équation des ondes, C. R. Acad. Sci. Paris. 302 (1986), $443-446$.

[10] A.E. Ingham, Some trigonometrical inequalities with applications to the theory of series, Math. Zeitschrift 41 (1936), 367-379.

[11] S. Jaffard, M. Tucsnak, E. Zuazua, On a theorem of Ingham, J. Fourier Anal. Appl. 3 (1997), 577-582.

[12] P. Jounieaux, Y. Privat, E. Trélat, Optimal boundary observation domain for the wave equation, ongoing work.

[13] C. Morawetz, Notes on time decay and scatteringfor some hyperbolic problems, Regional conference series in applied mathematics 19, SIAM, Philadelphia (1975).

[14] C. B. Morrey, On the Analyticity of the Solutions of Analytic Non-Linear Elliptic Systems of Partial Differntial Equations, American Journal of Mathematics, Vol. 80, No. 1 (Jan., 1958), pp. 198-237.

[15] K. Morris, Linear-quadratic optimal actuator location, IEEE Trans. Automat. Control 56 (2011), no. 1, $113-124$.

[16] E. Polak, Optimization, Algorithms and Consistent Approximations, Applied Mathematical Sciences, 124 Springer (1997).

[17] Y. Privat, M. Sigalotti, The squares of the Laplacian-Dirichlet eigenfunctions are generically linearly independent, ESAIM Control Optim. Calc. Var., Volume 16, Number 3, July-September 2010, pages 794-805.

[18] Y. Privat, E. Trélat, E. Zuazua, Optimal observability of the one-dimensional wave equation, J. Fourier Anal. Appl 19 (2013), no. 1, 514-544.

[19] Y. Privat, E. Trélat, E. Zuazua, Optimal observability of the multi-dimensional wave and Schrödinger equations in quantum ergodic domains, Preprint Hal (2013), 63 pages.

[20] Y. Privat, E. Trélat, E. Zuazua, Optimal shape and location of sensors or controllers for parabolic equations with random initial data, Preprint Hal (2013), 35 pages.

[21] M. Tucsnak, G. Weiss Observation and control for operator semigroups, Birkhäuser Advanced Texts: Basler Lehrbücher, Birkhäuser Verlag, Basel, Switzerland, 2009.

[22] A. Vande Wouwer, N. Point, S. Porteman, M. Remy, An approach to the selection of optimal sensor locations in distributed parameter systems, Journal of Process Control 10 (2000), 291-300.

[23] E. Zuazua, Controllability and observability of partial differential equations: Some results and open problems, Handbook of differential equations: evolutionary equations. Vol. III, 527-621. Elsevier/North-Holland, Amsterdam, 2007.

[24] A. Wächter, L.T. Biegler, On the implementation of an interior-point filter line-search algorithm for large-scale nonlinear programming, Mathematical Programming 106 (2006), 25-57. 